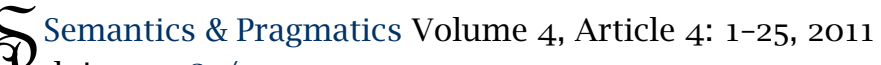

G doi: $10.3765 / \mathrm{sp} .4 .4$

\title{
Operators or restrictors? A reply to Gillies*
}

\author{
Justin Khoo \\ Yale University
}

Received 2011-04-24 / Accepted 2011-05-20 / Revision Received 2011-06-08 / Second Revision Received 2011-07-19 / Published 2011-07-26

\begin{abstract}
According to operator theories, if denotes a two-place operator. According to restrictor theories, if doesn't contribute an operator of its own but instead merely restricts the domain of some co-occurring quantifier. The standard arguments (Lewis 1975, Kratzer 1986) for restrictor theories have it that operator theories (but not restrictor theories) struggle to predict the truth conditions of quantified conditionals like
\end{abstract}

(1) a. If John didn't work at home, he usually worked in his office.

b. If John didn't work at home, he must have worked in his office.

Gillies (2010) offers a context-shifty conditional operator theory that predicts the right truth conditions for epistemically modalized conditionals like (1b), thus undercutting one standard argument for restrictor theories. I explore how we might generalize Gillies' theory to adverbially quantified conditionals like (1a) and deontic conditionals, and argue that a natural generalization of Gillies' theory - following his strategy for handling epistemically modalized conditionals - won't work for these other conditionals because a crucial assumption that epistemic modal bases are closed (used to neutralize the epistemic quantification contributed by if) doesn't have plausible analogs in these other domains.

Keywords: conditionals, modals, adverbial quantifiers

* Many thanks to Daniel Rothschild, two anonymous $S \& P$ referees, and members of the Yale Works in Progress seminar for extensive insightful comments and criticism that led to significant improvements. Extra special thanks to Kai von Fintel and Zoltán Szabó for numerous extremely helpful comments and discussion of earlier drafts.

(C)2011 Justin Khoo

This is an open-access article distributed under the terms of a Creative Commons NonCommercial License (creativecommons.org/licenses/by-nc/3.o). 
Back in the day, Lewis (1975) argued that no plausible conditional operator predicts the right truth conditions for adverbially quantified conditionals like: ${ }^{1}$

(2) a. Usually if John didn't work at home, he worked in his office.

b. If John didn't work at home, he usually worked in his office.

Suppose that if denotes the material conditional ' $\supset$ '. We have two choices for the scope relations between usually and ' $\supset$ ':

(3) a. Usually: (John didn’t work at home $\supset$ John worked in his office)

b. John didn’t work at home $\supset$ John usually worked in his office

But neither of (3a) or (3b) is equivalent to $(2 a) /(2 b)$. (3a) is true if John usually worked at home and (3b) is true if John usually worked in the office (even if his office was at home), though neither case is sufficient for the truth of (2a)/(2b). Rather both seem true iff

(4) Most times John didn't work at home, he worked in his office.

Kratzer (1986) noticed that Lewis's argument generalizes to the interaction of conditionals and modals as well. Consider an epistemically modalized conditional like

(5) If John drew a one-eyed King, he must have drawn a red card.

Since there is only one one-eyed King (the King of diamonds) in a standard deck of cards, (5) seems true. But suppose that if denotes ' $\supset$ '; then, as before, we have the two possible scope orders:

(6) a. Must: (John drew a one-eyed King $\supset$ John drew a red card)

b. John drew a one-eyed King $\supset$ John must have drawn a red card

(6a) is true if you (or the relevant party) are certain that John didn't draw a one-eyed King and (6b) is true if John didn't draw a one-eyed King. As before, neither condition is sufficient for the truth of (5), which instead requires knowing some additional fact (such as the fact about the deck mentioned

1 The placement of the adverb of quantification doesn't seem to affect the truth conditions: (2a) and (2b) seem equivalent. However, there are conditionals where the location of the adverb with respect to the conditional seems to result in truth conditional differences - see the examples in fn. 31 below, and Geurts 2004 for others. 
Operators or restrictors? A reply to Gillies

above). Indeed, (5) seems true iff

(7) It must be the case, given that John drew a one-eyed King, that he drew a red card.

In each of these conditionals (which I will call quantified conditionals), Lewis and Kratzer suggest that if doesn't denote an operator of its own, but rather restricts the domain of the co-occurring quantifier to elements of its domain that satisfy the if-clause. They predict that $(2 a) /(2 b)$ and (5) have the following structure:

(8) a. (Usually: John didn't work at home) John worked in his office

b. (Must: John drew a one-eyed King) John drew a red card

This "restrictor theory" is thus in a position to predict the correct truth conditions for conditionals with adverbial quantifiers or modals in their consequents, but it saddles us with difficult choices regarding bare conditionals (those which have no overt modal or quantifier) like

(9) If John didn't work at home, he worked in his office.

Since (9) apparently has no quantifier for the if-clause to restrict, restrictor theorists like Lewis and Kratzer face a dilemma: either if is ambiguous between a domain-restricting device and a conditional operator, or if restricts a covert (phonologically null) operator in bare conditionals. Lewis opts for the first horn (cf. Lewis 1973, 1975, 1976) whereas Kratzer opts for the second (cf. Kratzer 1986, 1991). Other things being equal, it would be better not to have to choose: a conditional operator theory that predicts correct truth conditions for both bare and quantified conditionals without resorting to lexical ambiguity would have a major theoretical advantage to a restrictor theory.

And perhaps we don't have to choose: Gillies's (2010) context-shifty conditional operator theory predicts correct truth conditions for both bare and epistemically modalized conditionals without resorting to lexical ambiguity or positing covert modals. But where the univocal restrictor theory has to posit a covert modal to account for the truth conditions of bare conditionals, Gillies' theory, being an operator theory, faces the opposite problem: it needs to neutralize the quantificational force of the conditional operator when an epistemic modal falls in its scope. Gillies (2010) handles this problem by holding that epistemic modal bases are closed (that they do not vary across 
epistemically possible worlds). The plausibility of this principle gives Gillies' theory an edge over restrictor theories, since their fix (covert modals or ambiguity) does not seem to have the same independent plausibility. ${ }^{2}$

Despite its success with epistemically modalized conditionals, it's not clear how to generalize Gillies' theory to handle those adverbially quantified conditionals that led Lewis to endorse a restrictor theory in the first place. In particular, the natural way of generalizing the theory to adverbs of quantification and deontic modals involves making an analogous assumption about the domains of those quantifiers, which leads to false empirical predictions. My claim is not that no context-shifty operator theory can handle the interaction between if and these other modals and quantifiers, but rather that one plausible way of extending the story - one taking Gillies's (2010) lead from epistemically modalized conditionals - doesn't seem to be the way to go. ${ }^{3}$

\section{Context-shifting conditional operators}

To illustrate Gillies' theory we'll focus on the second of three facts he argues any plausible theory of conditionals ought to predict:

Fact 2 (IF/MuST)

$$
\text { if } p, q \equiv \text { if } p \text {, must } q
$$

2 The emphasis on these theoretical concerns is mine, not Gillies'. He motivates his operator theory on primarily empirical grounds - that it does just as well as the restrictor theory with respect to epistemically modalized conditionals and better handles conditionals with conjunctive modal consequents. However, he also recognizes the theoretical benefits of not having to postulate covert modals (though he admits the costs of positing such a modal might be met), and assumes throughout that if is not lexically ambiguous (consider his statement of the problem for the non-context-shifty conditional operator theory, "it looks impossible to assign if the same meaning - thereby taking its contribution to be an iffy one - in all of our examples" (Gillies 2010: 23), and one of his concluding statements, "That is how if can mean the same iffy thing no matter whether the consequent is modal, and no matter the quantificational force of that modal, without running afoul of the Facts" (Gillies 2010: 39), emphasis mine).

3 The problems raised in this paper should also be of concern for the version of the shifty conditional story told by Yalcin (2007), who takes if to contribute an epistemic modal whose prejacent is evaluated relative to an information parameter which has been minimally changed to include the information of the if-clause. Although Yalcin doesn't address the interaction between if and modals/quantifiers, his theory will face the same problems raised here for Gillies' theory when it comes to predicting the right truth conditions for quantified conditionals. 
Operators or restrictors? A reply to Gillies

Here's an example (I won't argue for this fact here - see Gillies 2010: 14-15):

(10) a. If John didn't work at home, he worked in his office.

b. If John didn't work at home, he must have worked in his office.

Kratzer's univocal restrictor theory predicts IF/MUST by positing a covert modal in the logical form of if $p, q$ which has the same semantics as epistemic must, and holding that in each case if restricts that modal (and doesn't contribute a modal of its own). ${ }^{4}$ So, on a uniform restrictor theory like Kratzer's, both if $p, q$ and if $p$, must $q$ have a single modal which is restricted by if. Gillies' theory, being an operator theory, holds instead that if contributes a restricted epistemic necessity modal which takes its consequent as its nuclear scope. So, on Gillies' shifty operator theory, if $p$, must $q$ contains two modals - one contributed by if (the conditional operator) and one contributed by must. He predicts IF/MusT by appealing to the plausible assumption that epistemic modal bases are closed (they do not vary across epistemically accessible worlds) and holding that the consequent of a conditional is evaluated in a subordinate (shifted) context, which contains the information of the antecedent.

To put formally our foregoing informal discussion of Gillies' theory, we'll start by defining an ordinary (non-shifty) strict epistemic conditional operator, and then motivate the two key emendations Gillies makes: ${ }^{5}$

STRICT IFFINESS

$$
\llbracket i f_{E} p, q \rrbracket^{C, w}=1 \text { iff } \forall w^{\prime} \in\left(\llbracket \mathrm{E} \rrbracket^{C, w} \cap \llbracket p \rrbracket^{C}\right): \llbracket q \rrbracket^{C, w^{\prime}}=1
$$

4 Since Gillies assumes univocality, I'll set aside ambiguity theories for the rest of the paper to help focus the discussion. From here on, understand "the restrictor theory" as Kratzer's, which is univocal and appeals to covert modals to handle bare conditionals.

5 A comment about the formalism. «.』 is the semantic interpretation function, which assigns truth values to sentences relative to contexts $C$ and worlds $w$. The semantic value of $p$ in a context $C$ is a function from worlds to truth values (or alternatively, a set of worlds), which I will call a proposition. Thus, $\llbracket p \rrbracket^{C}=\left\{\mathrm{w}: \llbracket p \rrbracket^{C, w}=1\right\}$. $\llbracket \mathrm{E} \rrbracket^{C, w}$ is the set of epistemically accessible worlds compatible with the $C$-relevant information at $w$ (I will call this the modal base of the epistemic modal). I won't say anything about how this set is determined.

I am assuming that epistemic conditional operators and modals encode the function from contexts and worlds to modal bases as a covert element present in the logical form of sentences containing them, hence $i f_{E}$ and must $_{E}$. For perspicuity, I will only mark the subscript when introducing the semantics for these expressions, and omit it elsewhere unless it's needed to tell epistemic modals from deontic ones. 
Justin Khoo

Now, suppose that the must in (1ob) stays in situ, scoping under the conditional operator. ${ }^{6}$ We give the usual quantificational semantics to epistemic must:

$\llbracket$ must $_{E} p \rrbracket^{C, w}=1$ iff $\forall w^{\prime} \in \llbracket \mathrm{E} \rrbracket^{C, w}: \llbracket p \rrbracket^{C, w^{\prime}}=1$

Putting (11) and (12) together yields:

(13) StRICT IF/MUST

$$
\begin{array}{r}
\llbracket \text { if } p, \text { must } q \rrbracket^{C, w}=1 \text { iff } \forall w^{\prime} \in\left(\llbracket \mathrm{E} \rrbracket^{C, w} \cap \llbracket p \rrbracket^{C}\right): \\
\forall w^{\prime \prime} \in \llbracket \mathrm{E} \rrbracket^{C, w^{\prime}}: \llbracket q \rrbracket^{C, w^{\prime \prime}}=1
\end{array}
$$

The resulting truth conditions for epistemically modalized conditionals has two defects, both of which Gillies' theory avoids. The first defect is that quantified conditionals like (1ob) come out doubly quantified - the conditional operator quantifies over p-worlds compatible with the $C$-relevant information at $w$, and then must quantifies over worlds compatible with the $C$-relevant information at those worlds. However, this second layer of quantification gives rise to a counterexample to if $p, q \vDash$ if $p$, must $q .^{7}$

Gillies blocks counterexamples like this by holding that epistemic modal bases are closed: 8

\section{(14) Closed:}

$$
\text { If } w^{\prime} \in \llbracket \mathrm{E} \rrbracket^{C, w} \text { then } \llbracket \mathrm{E} \rrbracket^{C, w}=\llbracket \mathrm{E} \rrbracket^{C, w^{\prime}}
$$

6 In fact, Gillies (2010: §6) presents several arguments that this strict conditional operator gets the facts wrong for epistemically modalized conditionals like (1ob), no matter how strong the operator (what worlds it quantifies over) or where must scopes relative to it (notice his presumption that if is not ambiguous here), which I won't review here.

7 Suppose that $\llbracket \mathrm{E} \rrbracket^{C, w}=\left\{w, w_{1}\right\}, \llbracket \mathrm{E} \rrbracket^{C, w_{1}}=\left\{w_{1}, w_{2}\right\}, \llbracket p \rrbracket^{C}=\left\{w, w_{1}\right\}, \llbracket q \rrbracket^{C}=\left\{w, w_{1}\right\}$. Then $\forall w^{\prime} \in\left(\llbracket \mathrm{E} \rrbracket^{C, w} \cap \llbracket p \rrbracket^{C}\right): \llbracket q \rrbracket^{C, w^{\prime}}=1$, so $\llbracket$ if $p, q \rrbracket^{C, w}=1$. But notice $\exists w^{\prime} \in\left(\llbracket \mathrm{E} \rrbracket^{C, w} \cap \llbracket p \rrbracket^{C}\right)$ : $\exists w^{\prime \prime} \in \llbracket \mathrm{E} \rrbracket^{C, w^{\prime}}: \llbracket q \rrbracket^{C, w^{\prime \prime}}=0$; the witnessing world is $w_{1}$. Hence, on this model $\llbracket$ if $p$, must $q \rrbracket^{C, w}=0$.

8 This commitment is plausible, since Closed (also known as introspection) is entailed by

$$
\begin{array}{ll}
\text { a. } & w \in \llbracket \mathrm{E} \rrbracket^{C, w} \\
\text { b. } & \text { If } w^{\prime} \in \llbracket \mathrm{E} \rrbracket^{C, w} \text { then } \llbracket \mathrm{E} \rrbracket^{C, w} \subseteq \llbracket \mathrm{E} \rrbracket^{C, w^{\prime}}
\end{array}
$$

Reflexiveness

Euclideanness

both properties epistemic modal bases arguably have, given that epistemic modals express what is necessary/possible with respect to what is known (see von Fintel \& Gillies 2010 for further discussion, and Gillies 2010: 7 for the proof). It's worth pointing out that if reflexiveness isn't your bag (because you think must $p \notin p$ ), you can still get closed epistemic modal bases so long as you assume that they are euclidean and transitive. For our purposes, Closed is the crucial property - it is the weakest way to collapse stacked epistemic modals. 
Operators or restrictors? A reply to Gillies

That epistemic modal bases are closed disallows differences in what worlds are epistemically accessible across any set of epistemically accessible worlds. Given this principle, we can substitute $\llbracket \mathrm{E} \rrbracket^{C, w}$ for $\llbracket \mathrm{E} \rrbracket^{C, w^{\prime}}$ in (13) above to get

$$
\begin{aligned}
& \llbracket \text { if } p, \text { must } q \rrbracket^{C, w}=1 \text { iff } \forall w^{\prime} \in\left(\llbracket \mathrm{E} \rrbracket^{C, w} \cap \llbracket p \rrbracket^{C}\right): \\
& \forall w^{\prime \prime} \in \llbracket \mathrm{E} \rrbracket^{C, w}: \llbracket q \rrbracket^{C, w^{\prime \prime}}=1
\end{aligned}
$$

However, the resulting truth conditions are still problematic, for the initial quantification goes vacuous, yielding

$$
\llbracket \text { if } p, \text { must } q \rrbracket^{C, w}=1 \text { iff } \forall w^{\prime} \in \llbracket \mathrm{E} \rrbracket^{C, w}: \llbracket q \rrbracket^{C, w^{\prime}}=1
$$

which makes if $p$, must $q$ equivalent to must $q$ - an untenable result! Gillies' theory assumes Closed, and avoids this second problem by holding that the consequent of a conditional is not evaluated in the same context as the entire conditional; rather, it is evaluated in a different context in which the information of the antecedent is temporarily taken for granted, $C+p$ :

(17) SHIFTY IFFINESS

$\llbracket i f_{E} p, q \rrbracket^{C, w}=1$ iff $\forall w^{\prime} \in\left(\llbracket \mathrm{E} \rrbracket^{C, w} \cap \llbracket p \rrbracket^{C}\right): \llbracket q \rrbracket^{C+p, w^{\prime}}=1$

What is $C+p$ ? At this point, I don't have a general answer about the nature of update function + which delivers the subordinate context in which the consequent of the conditional is evaluated, ${ }^{9}$ but all that needs to be said at this point is how $C+p$ affects the function that delivers the modal base $\llbracket \mathrm{E} \rrbracket^{C, w}$ :

$$
\llbracket \mathrm{E} \rrbracket^{C+p}==_{\operatorname{def}} \lambda w \cdot \llbracket \mathrm{E} \rrbracket^{C, w} \cap \llbracket p \rrbracket^{C}
$$

Putting SHIFTY IfFINESS together with the semantics for must and scoping must under the conditional operator yields

GILLIES IF/MUST

$\llbracket$ if $p$, must $q \rrbracket^{C, w}=1$ iff $\forall w^{\prime} \in\left(\llbracket \mathrm{E} \rrbracket^{C, w} \cap \llbracket p \rrbracket^{C}\right): \llbracket$ must $q \rrbracket^{C+p, w^{\prime}}=1$

Gillies' theory is just SHIFTy IfFINESS + GILLIES IF/Must + Closed, and it supplies a uniform conditional operator that predicts the equivalence of if $p, q$

9 Gillies does, but I cannot follow his answer since he defines contexts as playing the role of modal bases for epistemic modals and conditionals. I assume that he will eventually say something more general on this point as well, if he is to extend his theory to account for adverbially quantified conditionals. 
and if $p$, must $q .{ }^{10}$ On this context-shifty semantics, if contributes a restricted epistemic modal whose prejacent (the consequent clause) is evaluated in a subordinate context. But equally crucial to Gillies' theory is Closed, which neutralizes the extra layer of epistemic quantification contributed by if. ${ }^{11}$ On the other hand, the univocal restrictor theory predicts IF/MUST without assuming Closed by positing a covert epistemic necessity modal in the bare conditional. ${ }^{12}$

So, both the restrictor theory and Gillies' shifty operator theory do equally well when it comes to predicting how if interacts with epistemic modals. But where the restrictor theory posits covert modals to capture the data, Gillies' theory instead appeals to the plausible principle that epistemic modal bases are closed; hence, it may claim this theoretical advantage. ${ }^{13}$ But also relevant to the evaluation of the theories is how they generalize to handle the interaction of if with adverbs of quantification and other kinds of modals. In the next two sections, I argue that a natural generalization of Gillies' theory (in line with the way Gillies himself suggests) to predict facts about the interaction of if with adverbs of quantification and deontic modals makes false empirical predictions.

10 Proof:

$\llbracket$ if $p$, must $q \rrbracket^{C, w}=1$ iff
a. $\quad \forall w^{\prime} \in\left(\llbracket \mathrm{E} \rrbracket^{C, w} \cap \llbracket p \rrbracket^{C}\right): \llbracket$ must $q \rrbracket^{C+p, w^{\prime}}=1$ iff
GILLIES IF/MuST
b. $\quad \forall w^{\prime} \in\left(\llbracket \mathrm{E} \rrbracket^{C, w} \cap \llbracket p \rrbracket^{C}\right): \forall w^{\prime \prime} \in \llbracket \mathrm{E} \rrbracket^{C+p, w^{\prime}}: \llbracket q \rrbracket^{C+p, w^{\prime \prime}}=1$ iff
TC must
c. $\forall w^{\prime} \in\left(\llbracket \mathrm{E} \rrbracket^{C, w} \cap \llbracket p \rrbracket^{C}\right): \forall w^{\prime \prime} \in\left(\llbracket \mathrm{E} \rrbracket^{C, w^{\prime}} \cap \llbracket p \rrbracket^{C}\right): \llbracket q \rrbracket^{C+p, w^{\prime \prime}}=1$ iff
d. $\forall w^{\prime} \in\left(\llbracket \mathrm{E} \rrbracket^{C, w} \cap \llbracket p \rrbracket^{C}\right): \forall w^{\prime} \in\left(\llbracket \mathrm{E} \rrbracket^{C, w} \cap \llbracket p \rrbracket^{C}\right): \llbracket q \rrbracket^{C+p, w^{\prime}}=1$ iff
Def. $\llbracket \mathrm{E} \rrbracket^{C+p}$
e. $\forall w^{\prime} \in\left(\llbracket \mathrm{E} \rrbracket^{C, w} \cap \llbracket p \rrbracket^{C}\right): \llbracket q \rrbracket^{C+p, w^{\prime}}=1$ iff
f. $\quad \llbracket$ if $p, q \rrbracket^{C, w}=1$
Closed
Vacuous Quantification
SHIFTY IFFINESS

11 Appeals to Closed are also present in the proofs that the context-shifty conditional operator accounts for the other two facts as well.

12 This is ensured by the covert modal $\square$ having the same truth conditions as epistemic must and both behaving the same with respect to if:

(i)
a. $\quad \llbracket$ if $p, \square q \rrbracket^{C, w}=1$ iff $\forall w^{\prime} \in\left(\llbracket \mathrm{E} \rrbracket^{C, w} \cap \llbracket p \rrbracket^{C}\right): \llbracket q \rrbracket^{C, w^{\prime}}=1$
b. $\quad \llbracket$ if $p$, must $q \rrbracket^{C, w}=1$ iff $\forall w^{\prime} \in\left(\llbracket \mathrm{E} \rrbracket^{C, w} \cap \llbracket p \rrbracket^{C}\right): \llbracket q \rrbracket^{C, w^{\prime}}=1$

13 In addition, Gillies claims an empirical advantage over the restrictor theory, since he argues that the operator theory fares better when it comes to handling conditionals with conjunctive modal consequents. I am putting aside this data for now. 
Operators or restrictors? A reply to Gillies

\section{Adverbially quantified conditionals}

Though he recognizes the need to generalize his theory to adverbs of quantification, Gillies explicitly sets aside treating their interaction with if as an "argument for another day". However, he gives us a hint about how to extend his theory to account for them: "First, adjust the kinds of information represented by the context so that we can sensibly quantify over individuals and the events they participate in. Second, allow that quantificational domains can be restricted by material in if-clauses - those domains play the role of the subordinate or derived context. Adverbs of quantification appear under the conditional and have their usual denotations" (Gillies 2010: 31). Let's adjust first. Adverbs of quantification demand quantification over things that are "smaller" than worlds, and there are two main ways to handle this in the literature. ${ }^{14}$ Since deciding between them is not our purpose here, I will simply assume the situation based approach. ${ }^{15}$ Moving to situation semantics necessitates changing our interpretation function slightly: it now assigns truth values to sentences relative to a context $C$ and a situation $s$ instead of a world (hence, propositions are treated as sets of situations now). I make the following (standard - see Kratzer 1989, von Fintel 2004) assumptions about situations and worlds:

i. Worlds are maximal situations: situations which are not part of any other situation.

ii. Every situation is part of exactly one world.

iii. Natural language propositions are persistent: for all propositions $p$ and any situations $s, s^{\prime}: s$ is a part of $s^{\prime}$, if $\llbracket p \rrbracket^{C, s}=1$ then $\llbracket p \rrbracket^{C, s^{\prime}}=1$.

Nothing crucial about our semantics for epistemic modals or conditionals needs changing, given our assumption that each situation is part of exactly one world. We'll occasionally need to talk about the world of a particular situation, for which I use the following shorthand:

(20) $\quad w_{s}==_{\text {def }}$ the world of $s$

14 Quantification over cases/variable assignments, (e.g. Lewis 1975, Kamp 1981, Heim 1982, Chierchia 1992) or quantification over situations, understood as parts of worlds, (e.g. Berman 1987, Kratzer 1989, 2011b, Heim 1990, von Fintel 1994, 2004, Elbourne 2005).

15 Nothing crucial turns on this assumption, since the problem for extending Gillies' theory isn't a limitation of one kind of semantics but a general problem of neutralizing the extra layer of epistemic quantification. 
Justin Khoo

Then we may recast our semantics of must and Gillies' semantics for if as follows: ${ }^{16}$

(21) $\llbracket$ must $_{E} p \rrbracket^{C, s}=1$ iff $\forall s^{\prime} \in \llbracket \mathrm{E} \rrbracket^{C, w_{s}}: \llbracket p \rrbracket^{C, s^{\prime}}=1$

(22) SHIFTY IFFINESS ${ }^{17}$

$\llbracket$ if $f_{E} p, q \rrbracket^{C, s}=1$ iff $\forall s^{\prime} \in\left(\llbracket \mathrm{E} \rrbracket^{C, w_{s}} \cap \llbracket p \rrbracket^{C}\right): \llbracket q \rrbracket^{C+p, s^{\prime}}=1$

Our new semantics requires a minor (notational, given our assumptions) change to our definition of $\llbracket \mathrm{E} \rrbracket^{C+p}$ :

$$
\llbracket \mathrm{E} \rrbracket^{C+p}==_{\operatorname{def}} \lambda s . \llbracket \mathrm{E} \rrbracket^{C, w_{s}} \cap \llbracket p \rrbracket^{C}
$$

We assign the following truth conditions to usually $p^{: 18}$

(24) $\llbracket$ usually $_{A} p \rrbracket^{C, s}=1$ iff most $s^{\prime} \in \llbracket \mathrm{A} \rrbracket^{C, s}: \llbracket p \rrbracket^{C, s^{\prime}}=1$

Here, we let $\llbracket \mathrm{A} \rrbracket^{C, s}$ play the role of the "modal base" of the situation quantifier - it's just a set of contextually restricted situations - let's call it the adverbial base. ${ }^{19}$ The truth conditions for other adverbial quantifiers are generated by varying the force of the quantifier they encode: always is universal quantification, sometimes is existential quantification, and so on. These truth conditions are close enough for our purposes (setting aside the complications mentioned in fn 18) to serve as the "usual" denotations of adverbial quantifiers (in situation semantics).

The foregoing semantics also allows for an easy statement of the restrictor theory truth conditions of if $p$, usually $q:^{20}$

\section{(25) RESTRICTOR IF/USUALLY}

$\llbracket$ if $p$, usually $q \rrbracket^{C, s}=1$ iff most $s^{\prime} \in\left(\llbracket \mathrm{A} \rrbracket^{C, s} \cap \llbracket p \rrbracket^{C}\right): \llbracket q \rrbracket^{C, s^{\prime}}=1$

16 Since worlds are maximal situations, we can assume (to keep the change to situation semantics to a minimum) that $\forall s^{\prime} \in \llbracket \mathrm{E} \rrbracket^{C, w_{s}}: s^{\prime}$ is a world. This means Closed does not need to be changed either.

17 Understand SHIFTY IFFINESS as referring to this shifty situation semantics for if for this section only. Everywhere else in the paper it refers to the semantics given in (17).

18 Ignoring complications of extensions of situations and minimal situations which don't concern us: see von Fintel 2004, Kratzer 2011b, Portner 2009 for discussion.

19 As before, I assume that the function from contexts and situations to adverbial bases is part of the logical form of sentences containing adverbs of quantification-hence, usually $y_{A}$ I omit the subscript for perspicuity from here on. How context determines adverbial bases is beyond the scope of this paper. See von Fintel 2004, Beaver \& Clark 2008 for some proposals. 20 I won't give a compositional derivation of the restrictor theory's truth conditions here. See Farkas \& Sugioka 1983 or Kratzer 2011a for a compositional implementation. 
Operators or restrictors? A reply to Gillies

Before we see what truth conditions the extended Gillies' theory assigns to if $p$, usually $q$, we must address what it is for the domains of adverbial quantifiers to "play the role of the subordinate or derived context". This amounts to explaining how $C+p$ affects adverbial bases. I'm not sure how to proceed here except to mimick Gillies' treatment of how $C+p$ affects epistemic modal bases: ${ }^{21}$

$$
\llbracket \mathrm{A} \rrbracket^{C+p}={ }_{\operatorname{def}} \lambda s \cdot \llbracket \mathrm{A} \rrbracket^{C, s} \cap \llbracket p \rrbracket^{C}
$$

Finally, combining SHIFTY IFFINESS with the semantics for usually and (as Gillies suggests) scoping the adverbial quantifier under the conditional operator yields the following truth conditions for if $p$, usually $q$ :

$$
\begin{aligned}
& \text { GILLIES IF/UsuALLY } \\
& \begin{aligned}
\llbracket \text { if } p \text {, usually } q \rrbracket^{C, s}=1 \text { iff } \forall s^{\prime} & \in\left(\llbracket \mathrm{E} \rrbracket^{C, w_{s}} \cap \llbracket p \rrbracket^{C}\right): \\
& \text { most } s^{\prime \prime} \in\left(\llbracket \mathrm{A} \rrbracket^{C, s^{\prime}} \cap \llbracket p \rrbracket^{C}\right): \llbracket q \rrbracket^{C+p, s^{\prime \prime}}=1
\end{aligned}
\end{aligned}
$$

Notice that the part underneath the universal quantifier over epistemically possible $p$-worlds is equivalent (assuming no modals in $q$ ) to the restrictor theory truth conditions for if $p$, usually $q$. Since if-shifted contexts are "inherited" left-to-right, we evaluate usually $q$ in $C+p$. This is the same mechanism that, along with the assumption of Closed, allows GILLIES IF/MUST to predict the right truth conditions for if $p$, must $q$. But now return to the fact that,

21 This is a promising strategy because, like the restrictor theory, it treats epistemically modalized conditionals and adverbially quantified conditionals in like fashion, which amounts to a very clean theory. However, proceeding in this way puts a constraint on how we understand + , now that we've moved from epistemic modals to adverbial quantifiers. If we understand + as a temporary form of assertion - such that asserting that $p$ in context $C$ results in the updated context $C+p-(26)$ would entail that the following sentence is inconsistent:

(i) John is at home, though he usually isn't at home.

Since usually would quantify over only situations in which John is at home. Of course, (i) is perfectly acceptable, unlike

(ii) ??John is at home, though he probably isn't at home.

So it must be that +-updated contexts affect adverbial bases while assertion-updated contexts do not. Incorporating this insight would involve telling a full story about how context constrains adverbial bases. I won't go into the possible ways to do this here, but merely point out this further issue for extending Gillies' theory in a uniform way to handle adverbial quantifiers. Thanks to Daniel Rothschild for bringing this issue to my attention and encouraging me to say more here. 
for GILLIES IF/USUALLY, the usually $q$ which occurs in if $p$, usually $q$ falls under a universal quantifier over epistemically possible worlds. Hence, the GILLIES IF/UsUALLY truth conditions are stronger than those of RESTRICTOR IF/USUALLY: on the latter, if $p$, usually $q$ requires for its truth that all $p$-worlds compatible with the relevant knowledge or evidence be ones at which most (relevant) $p$-situations are $q$-situations. ${ }^{22}$

Our target reading for if $p$, usually $q$ is the one that motivated Lewis and Kratzer to adopt restrictor theories in the first place, which (following Geurts 2004) I'll call the O-reading of adverbially quantified conditionals - the Oreading of if $p$, usually $q$ may be paraphrased as "most (relevant) $p$-situations are $q$-situations". However, in addition to the O-reading, Geurts identifies another reading carried by many quantified conditionals, which he calls the C-reading - the C-reading of if $p$, usually $q$ may be paraphrased as "if it turns out that $p$, then usually $q "{ }^{23}$ As it stands, GiLlies IF/UsUALLY does not predict the O-reading of if $p$, usually $q$-its O-reading means roughly that most $p$-situations are $q$-situations, while GILLIES IF/USUALLY predicts it means rather that it is epistemically necessary given $p$ that most $p$-situations are $q$-situations. The trouble facing GILLIES IF/USUALLY is analogous to the problem that GILLIES IF/MUST predicts that epistemically modalized conditionals end up doubly quantified. Recall that to handle the earlier problem, Gillies' theory appealed to Closed to neutralize the epistemic quantification contributed by if when an epistemic modal falls in its scope. Hence, to

22 To demonstrate that the GILLIES IF/UsuALLY truth conditions are stronger than the RESTRICTOR IF/UsUALLY truth conditions, here's a scenario in which the latter are satisfied but the former not. Suppose $\llbracket \mathrm{E} \rrbracket^{C, w_{s}}=\left\{w_{s}, w_{t}\right\}$, $\llbracket \mathrm{A} \rrbracket^{C+p, w_{t}}=\{t\}$, $\llbracket \mathrm{A} \rrbracket^{C, s}=\{s, u, v\}$, and that $\llbracket p \rrbracket^{C}=$ $\left\{s, t, u, v, w_{s}, w_{t}, w_{u}, w_{v}\right\}$ and $\llbracket q \rrbracket^{C}=\left\{s, u, w_{s}, w_{u}\right\}$, and hence $\llbracket q \rrbracket^{C+p}=\left\{s, u, w_{s}, w_{u}\right\}$. On this scenario, $\exists s^{\prime} \in\left(\llbracket \mathrm{E} \rrbracket^{C, w_{s}} \cap \llbracket p \rrbracket^{C}\right)$ : most $s^{\prime \prime} \in \llbracket \mathrm{A} \rrbracket^{C+p, s^{\prime}}: \llbracket q \rrbracket^{C, s^{\prime \prime}}=0$ - the situation is $w_{t}$. However, most $s^{\prime} \in\left(\llbracket \mathrm{A} \rrbracket^{C, s} \cap \llbracket p \rrbracket^{C}\right): \llbracket q \rrbracket^{C, s^{\prime}}=1$ because $s, u \in \llbracket q \rrbracket^{C}$, even though $v$ is not.

23 Some adverbially quantified conditionals turn out to be ambiguous between the two readings. In a null context, the O-reading of

(i) If John didn't work at home, he usually worked in his office.

is prevalent, though its C-reading is dominant in the following context:

(ii) Last year, John either worked at home, or he usually worked in his office. So, if John didn't work at home, he usually worked in his office.

My focus in this paper is entirely on the O-readings of adverbially quantified conditionals and on the problems with extending Gillies' theory to capture them. We'll see there are problems for extending Gillies' theory to capture C-readings in fn31. 
Operators or restrictors? A reply to Gillies

predict the target reading of if $p$, usually $q$, the most natural fix for this extension of Gillies' theory would be to appeal to an analogous principle to neutralize the epistemic quantification contributed by if when an adverbial quantifier appears in its scope. Closed disallows differences in epistemic modal bases across epistemically accessible worlds. In the adverbial case, the analogous principle is one that disallows differences in adverbial bases across epistemically possible worlds:

\section{Closed*}

For all situations $s, t$, if $w_{t} \in \llbracket \mathrm{E} \rrbracket^{C, w_{s}}$ then $\llbracket \mathrm{A} \rrbracket^{C, s}=\llbracket \mathrm{A} \rrbracket^{C, t}$

Assuming Closed* would allow Gillies to neutralize the extra epistemic quantification contributed by if when an adverb of quantification appears underneath it - the resulting theory predicts the target reading of if $p$, usually $q .{ }^{24}$ But now the theory faces a new problem, for this principle isn't nearly as plausible as Closed. For one, it doesn't seem to follow from any plausible features of epistemic or adverbial bases. More importantly, the combination of GILLIES IF/MusT + Closed + Closed* predicts that conditionals of the form if $p$, must usually $q$ are true iff most (relevant) $p$-situations are $q$-situations (i.e., that they have the same truth conditions as the target O-reading of if $p$, usually q), ${ }^{25}$ but this is false, as I will show below. Thus, ensuring GILLIES

24 Proof:

(i) $\llbracket$ if $p$, usually $q \rrbracket^{C, s}=1$ iff
a. $\forall s^{\prime} \in\left(\llbracket \mathrm{E} \rrbracket^{C, w_{s}} \cap \llbracket p \rrbracket^{C}\right)$ : most $s^{\prime \prime} \in \llbracket \mathrm{A} \rrbracket^{C+p, s^{\prime}}: \llbracket q \rrbracket^{C+p, s^{\prime \prime}}=1$ iff GILLIES IF/UsuALLY
b. $\forall s^{\prime} \in\left(\llbracket \mathrm{E} \rrbracket^{C, w_{s}} \cap \llbracket p \rrbracket^{C}\right):$ most $s^{\prime \prime} \in\left(\llbracket \mathrm{A} \rrbracket^{C, s^{\prime}} \cap \llbracket p \rrbracket^{C}\right): \llbracket q \rrbracket^{C+p, s^{\prime \prime}}=1$ iff Def. $\llbracket \mathrm{A} \rrbracket^{C+p}$
c. $\quad \forall s^{\prime} \in\left(\llbracket \mathrm{E} \rrbracket^{C, w_{s}} \cap \llbracket p \rrbracket^{C}\right):$ most $s^{\prime} \in\left(\llbracket \mathrm{A} \rrbracket^{C, s} \cap \llbracket p \rrbracket^{C}\right): \llbracket q \rrbracket^{C+p, s^{\prime}}=1$ iff $\quad$ Closed*
d. $\quad$ most $s^{\prime} \in\left(\llbracket \mathrm{A} \rrbracket^{C, s} \cap \llbracket p \rrbracket^{C}\right): \llbracket q \rrbracket^{C+p, s^{\prime}}=1$
Vacuous Quantification

25 Proof:

(i) $\quad$ if $p$, must usually $q \rrbracket^{C, s}=1$ iff
a. $\quad \forall s^{\prime} \in\left(\llbracket \mathrm{E} \rrbracket^{C, w_{s}} \cap \llbracket p \rrbracket^{C}\right): \llbracket$ must usually $q \rrbracket^{C+p, s^{\prime}}=1$ iff $\quad$ GILLIES IF/MusT
b. $\quad \forall s^{\prime} \in\left(\llbracket \mathrm{E} \rrbracket^{C, w_{s}} \cap \llbracket p \rrbracket^{C}\right): \forall s^{\prime \prime} \in \llbracket \mathrm{E} \rrbracket^{C+p, w_{s^{\prime}}}: \llbracket$ usually $q \rrbracket^{C+p, s^{\prime \prime}}=1$ iff $\quad$ TC must
c. $\forall s^{\prime} \in\left(\llbracket \mathrm{E} \rrbracket^{C, w_{s}} \cap \llbracket p \rrbracket^{C}\right): \forall s^{\prime \prime} \in\left(\llbracket \mathrm{E} \rrbracket^{C, w_{s^{\prime}}} \cap \llbracket p \rrbracket^{C}\right): \llbracket$ usually $q \rrbracket^{C+p, s^{\prime \prime}}=1$ iff $\quad$ Def. $\llbracket \mathrm{E} \rrbracket^{C+p}$
d. $\forall s^{\prime} \in\left(\llbracket \mathrm{E} \rrbracket^{C, w_{s}} \cap \llbracket p \rrbracket^{C}\right): \forall s^{\prime} \in\left(\llbracket \mathrm{E} \rrbracket^{C, w_{s}} \cap \llbracket p \rrbracket^{C}\right): \llbracket$ usually $q \rrbracket^{C+p, s^{\prime}}=1$ iff $\quad$ Closed
e. $\forall s^{\prime} \in\left(\llbracket \mathrm{E} \rrbracket^{C, w_{s}} \cap \llbracket p \rrbracket^{C}\right): \llbracket$ usually $q \rrbracket^{C+p, s^{\prime}}=1 \quad$ Vacuous Quantification
f. $\forall s^{\prime} \in\left(\llbracket \mathrm{E} \rrbracket^{C, w_{s}} \cap \llbracket p \rrbracket^{C}\right):$ most $s^{\prime \prime} \in \llbracket \mathrm{A} \rrbracket^{C+p, s^{\prime}}: \llbracket q \rrbracket^{C+p, s^{\prime \prime}}=1$ iff $\quad$ TC usually $q$
g. $\forall s^{\prime} \in\left(\llbracket \mathrm{E} \rrbracket^{C, w_{s}} \cap \llbracket p \rrbracket^{C}\right):$ most $s^{\prime \prime} \in\left(\llbracket \mathrm{A} \rrbracket^{C, s^{\prime}} \cap \llbracket p \rrbracket^{C}\right): \llbracket q \rrbracket^{C+p, s^{\prime \prime}}=1$ iff Def. $\llbracket \mathrm{A} \rrbracket^{C+p}$
h. $\forall s^{\prime} \in\left(\llbracket \mathrm{E} \rrbracket^{C, w_{s}} \cap \llbracket p \rrbracket^{C}\right):$ most $s^{\prime} \in\left(\llbracket \mathrm{A} \rrbracket^{C, s} \cap \llbracket p \rrbracket^{C}\right): \llbracket q \rrbracket^{C+p, s^{\prime}}=1$ iff $\quad$ Closed*
i. $\quad$ most $s^{\prime} \in\left(\llbracket \mathrm{A} \rrbracket^{C, s} \cap \llbracket p \rrbracket^{C}\right): \llbracket q \rrbracket^{C+p, s^{\prime}}=1$ iff
j. $\quad \llbracket$ if $p$, usually $q \rrbracket^{C, s}=1$
Vacuous Quantification GILLIES IF/USUALLY 
IF/UsUALLY predicts the right truth conditions for if $p$, usually $q$ by assuming Closed* won't work - the theory predicts the right truth conditions for if $p$, usually $q$ at the expense of predicting the wrong truth conditions for if $p$, must usually $q$. Hence, the analogous solution to an analogous problem (the combination of if/must vs. the combination of if/usually) for Gillies' theory fails.

To show what's wrong with the predicted truth conditions for if $p$, must usually $q$ I will review several instances of conditionals of that form. Let's divide the choice of if-clause into those felicitously replaceable by a whenclause, and those which are not. ${ }^{26}$ Begin with an example of the former:

(29) If John goes to San Antonio, he must usually visit the Alamo.

Intuitively, (29) means something like "it must be the case that John usually visits the Alamo if he goes to San Antonio" - it is used to express the claim that it follows from some knowledge or evidence that most situations in which John goes to San Antonio are situations in which he visits the Alamo (a favorable context is one in which it's common knowledge that John is an avid scholar of the Texas Revolution). Notice that (29) does not express the non-modalized claim that most situations in which John goes to San Antonio are situations in which he visits the Alamo; rather, this claim is expressed by the O-reading of

(30) If John goes to San Antonio, he usually visits the Alamo.

(29) is a claim about what follows from some knowledge or evidence, while (30) is a claim about what John usually does in San Antonio. Thus, where the if-clause is felicitously replaceable by a when-clause, as in (29), it's not the case that if $p$, must usually $q$ is true iff most (relevant) $p$-situations are $q$-situations, contrary to the prediction of GILLIES IF/MusT + Closed + Closed*.27

Next, consider those if-clauses which are not felicitously replaceable by when-clauses. There seem to be three kinds of such if-clauses: statives,

26 Some if-clauses are ambiguous between readings in which the replacement is felicitous and ones in which it isn't. The reader is invited to run those through both tests.

27 GILLIES IF/UsUALLY might predict the correct reading for (29) if it allowed must to scope over the entire adverbially quantified conditional. The point here is that, as stated, the theory interprets must underneath if-clauses, and thus wrongly predicts a reading of (29) on which it is equivalent to the O-reading of (30). 
Operators or restrictors? A reply to Gillies

specific eventives, and habituals. Here are examples of each, respectively:

(31) a. If John is a basketball legend, he must usually attend home games.

b. If John wasn't home by 8pm last night, he must usually work late.

c. If John typically worked at home last year, he must usually go out for dinner.

None of these are true iff most (relevant) $p$-situations are $q$-situations. Each of (31a), (31b), and (31c) are claims about what follows from some evidence or knowledge, which the truth conditions predicted by GILLIES IF/MUST + Closed + Closed* don't reflect. Notice also that the O-reading (our target reading, the reading that's true iff most (relevant) $p$-situations are $q$-situations) of if $p$, usually $q$ stays the same when we "raise" usually over if - witness the equivalence (at least in O-reading) of

(32) a. If John doesn't work at home, he usually works in his office.

b. Usually if John doesn't work at home, he works in his office.

Thus, since (the O-reading of) if $p$, usually $q$ is true iff usually if $p, q$ is true iff most (relevant) $p$-situations are $q$-situations, we can test to see whether (31a), (31b) and (31c) are true iff most (relevant) $p$-situations are $q$-situations by seeing if each is equivalent to their usually if $p, q$ counterpart:

(33) a. ??Usually if John is a basketball legend, he attends home games.

b. ??Usually if John wasn't home by 8 pm last night, he works late.

c. ??Usually if John typically worked at home last year, he goes out for dinner.

Notice that, unlike (31a), (31b), and (31c), their usually if $p, q$ counterparts are infelicitous. This is evidence that none of (31a), (31b) or (31c) are equivalent to their usually if $p, q$ counterparts and thus that none of them are true iff most (relevant) $p$-situations are $q$-situations (for their respective $p$ s and $q$ s).

In addition to intuitions about what each are about and the infelicity of their usually if $p, q$ counterparts, there are counterexamples to the predicted truth conditions for (31a), (31b), and (31c). For the sake of space, I will illustrate just one. Take (31c) and consider the following scenario: you and a friend are discussing John's daily habits. Neither of you are sure where he typically worked last year or where he usually eats dinner, but you have evidence that rules out possibilities in which he typically worked at home last year and doesn't usually go out for dinner (suppose your evidence entails 
that people who typically worked at home last year usually go out for dinner), so you say (31c). What you've said in this context is true since your evidence plus the assumption that John typically worked at home last year entails that he usually goes out for dinner. But notice that in articulating this scenario I've said nothing about whether most situations in which John typically worked at home last year are ones in which John goes out for dinner. Thus, it may even be true that John never actually goes out for dinner - this may be true in this scenario as long as John actually didn't typically work at home last year $^{28}$ - and thus that no situations in which John typically worked at home last year are ones in which he goes out for dinner. Hence, (31c) may be true even though the truth conditions predicted by GILLIES IF/MUST + Closed + Closed* are unsatisfied.

Here's the problem, in sum. To predict the facts discussed in Gillies 2010, Gillies must neutralize the epistemic quantification contributed by if when an epistemic modal appears underneath it; he does so by assuming that epistemic modal bases do not vary across epistemically possible worlds (Closed). Likewise, the extended theory needs a way to neutralize the epistemic quantification contributed by if when an adverbial quantifier appears under it in order to predict the O-reading of if $p$, usually $q$. The analogous fix would be to adopt the principle that adverbial bases do not vary across epistemically possible worlds (Closed*). But while GILLIES IF/UsUALLY + Closed* predicts the O-reading of if $p$, usually $q$, GILLIES IF/MUST + Closed + Closed* neutralizes both the conditional operator and epistemic must, leaving usually to be evaluated in the if-shifted subordinate context, thus predicting that if $p$, must usually $q$ is true iff most $p$-situations are $q$-situations, which I've

28 This assumption ensures that the evaluation world is not among the worlds being quantified over by must in (31c). More formally, where $s$ is the evaluation situation, suppose that $\llbracket \mathrm{E} \rrbracket^{C, w_{s}} \cap \llbracket J o h n$ typically worked at home last year $\rrbracket^{C}=\{t\}$ and that $\llbracket J o h n$ usually goes out for dinner $\rrbracket^{C, t}=1$. Thus, $\forall s^{\prime} \in\left(\llbracket \mathrm{E} \rrbracket^{C, w_{s}} \cap \llbracket J o h n\right.$ typically worked at home last year $\left.\rrbracket^{C}\right): \llbracket J o h n$ usually goes out for dinner $\rrbracket^{C, s^{\prime}}=1$ and intuitively, (31c) is true (since the relevant knowledge plus the assumption that John typically worked at home last year entails that John usually goes out for dinner). But it's compatible with this that $\llbracket J o h n$ never goes out for dinner $\rrbracket^{C, S}=$ 1 , that is, $\neg \exists s^{\prime} \in \llbracket \mathrm{A} \rrbracket^{C, s}: \llbracket J o h n$ goes out for dinner $\rrbracket^{C, s^{\prime}}=1$. And hence, for any $p$, it's not the case that most $s^{\prime} \in\left(\llbracket \mathrm{A} \rrbracket^{C, s} \cap \llbracket p \rrbracket^{C}\right): \llbracket J o h n$ goes out for dinner $\rrbracket^{C, s^{\prime}}=1$. 
Operators or restrictors? A reply to Gillies

argued is incorrect. ${ }^{29,30}$

The problem isn't avoided by pointing out that there is another reading many adverbially quantified conditionals carry, Geurts' C-reading, which GILliES IF/UsUALLY (by itself, without Closed*) might seem to predict for if $p$, usually $q$. The problem for Gillies' theory (and any univocal epistemic conditional operator theory) is how to predict the O-reading of if $p$, usually $q$ without also predicting the wrong truth conditions for if $p$, must usually $q$. That if $p$, usually $q$ carries another reading that the theory predicts won't help with this original problem. ${ }^{31}$

29 A related problem concerns extending Gillies' theory to handle those bare conditionals which are equivalent to an adverbially quantified conditional:

a. If John doesn't work at home, he works in his office. $\equiv$

b. If John doesn't work at home, he typically works in his office.

Bare conditionals like (ia) have been called "multi-case" conditionals by Kadmon (1987) and are treated explicitly as restrictors over a covert adverbial quantifier in Farkas \& Sugioka (1983) (see also Krifka et al. (1995) for evidence that simple habitual sentences like John walks to school contain a covert adverbial quantifier). The argument in this section shows that an analogous approach (context-shifting conditional operator over a covert adverbial quantifier) to these conditionals is not available to the natural extension of Gillies' theory - instead, such a theory must posit two different kinds of conditional operators to handle these different kinds of bare conditionals, and hence, must be non-univocal.

30 The restrictor theory predicts the right truth conditions for if $p$, must usually $q$ as long as it holds that the if-clause restricts must instead of usually:

$$
\llbracket \text { if } p \text {, must usually } q \rrbracket^{C, s}=1 \text { iff } \forall s^{\prime} \in\left(\llbracket \mathrm{E} \rrbracket^{C, w_{s}} \cap \llbracket p \rrbracket^{C}\right): \text { most } s^{\prime \prime} \in \llbracket \mathrm{A} \rrbracket^{C, s^{\prime}}: \llbracket q \rrbracket^{C, s^{\prime \prime}}=1
$$

31 Furthermore, there are reasons to think that GILliEs If/UsuALly by itself (without Closed*) doesn't predict the C-reading of if p, usually q. As Geurts (2004) points out, the O-/Cambiguity is often subject to the placement of the adverb of quantification with respect to the if-clause, which explains the difference in felicity between

(i) a. If John worked at home last night, he usually goes out for dinner.

b. ??Usually if John worked at home last night, he goes out for dinner.

Adverbial quantifiers generally dislike being restricted to a single element, which is what the if-clause (being specific eventive) in (ib) tries to do; since there is no other reading it carries, it is infelicitous. However, (ia) carries the additional C-reading in which the if-clause doesn't restrict usually and, because of the infelicity of the O-reading, we prefer the felicitous C-reading (notice we can add must to the consequent of (ia) without changing its meaning). On the context-shifty theory, restriction-via-if-clause is done by context-shifting - this is how Gillies is able to mimic epistemic must being restricted by if. But notice that GILLIES IF/UsuALlY predicts that usually in (ia) is evaluated in the shifted context (shifted contexts 
Furthermore, although Gillies hints that in extending his theory to adverbial quantifiers we should scope them under the conditional operator, one might try to sidestep the problem altogether by rejecting GILLIES IF/USUALLY and instead scope the adverbial quantifier over the conditional operator, yielding usually (if $p, q$ ). Aside from being ad hoc - there doesn't seem to be any independent reason for treating the scopal properties of modals and adverbials differently - it yields in the wrong results:

$$
\begin{array}{r}
\llbracket \text { usually (if } p, q) \rrbracket^{C, s}=1 \text { iff most } s^{\prime} \in \llbracket \mathrm{A} \rrbracket^{C, s}: \forall s^{\prime \prime} \in\left(\llbracket \mathrm{E} \rrbracket^{C, w_{s^{\prime}}} \cap \llbracket p \rrbracket^{C}\right): \\
\llbracket q \rrbracket^{C+p, s^{\prime \prime}}=1
\end{array}
$$

This may be paraphrased by, in most situations, it is epistemically necessary given $p$ that $q$, and certainly isn't the O-reading of if $p$, usually $q .{ }^{32}$

Summing up, adverbially quantified conditionals like if $p$, usually $q$ carry O-readings which are true iff most (relevant) $p$-situations are $q$-situations, whereas it is not the case that conditionals like if $p$, must usually $q$ are true iff most (relevant) $p$-situations are $q$-situations. To predict both of these facts, Gillies' theory needs a way to neutralize the epistemic quantification contributed by if when an adverbial quantifier falls in its scope without also neutralizing the epistemic quantification contributed by the must scoping between the conditional operator and adverbial quantifier in conditionals of the form

\section{(35) if $p$, must ADVQ $q$}

are inherited left-to-right) and hence should be as infelicitous as (ib) (since on both usually ends up restricted to a single element). But it is not. Thus, it seems doubtful that GiLLIES If/Usually (by itself, without Closed*) predicts the C-reading of adverbially quantified conditionals.

32 An explicit counterexample: let $\llbracket \mathrm{E} \rrbracket^{C, w_{s}}=\left\{w_{s}, w_{t}, w_{u}, w_{v}\right\}$, $\llbracket \mathrm{E} \rrbracket^{C, w_{u}}=\left\{w_{s}, w_{t}, w_{u}, w_{v}\right\}$, $\llbracket \mathrm{A} \rrbracket^{C, s}=\{s, u, v\}, \llbracket p \rrbracket^{C}=\left\{s, u, v, w_{s}, w_{u}, w_{v}\right\}, \llbracket q \rrbracket^{C}=\left\{s, u, w_{s}, w_{u}\right\}$ (and hence, $\llbracket q \rrbracket^{C+p}$ $\left.=\left\{s, u, w_{s}, w_{u}\right\}\right)$. Then, for most $s^{\prime} \in \llbracket \mathrm{A} \rrbracket^{C, s}: \exists s^{\prime \prime} \in\left(\llbracket \mathrm{E} \rrbracket^{C, w_{s^{\prime}}} \cap \llbracket p \rrbracket^{C}\right): \llbracket q \rrbracket^{C+p, s^{\prime \prime}}=\mathrm{o}$, the situations are $s$ and $u$, and the falsifying-q situation is $w_{v}$. Hence,

(i) $\llbracket$ usually (if $p, q) \rrbracket^{C, s}=\mathrm{o}$

But since most $s^{\prime} \in\left(\llbracket \mathrm{A} \rrbracket^{C, s} \cap \llbracket p \rrbracket^{C}\right): \llbracket q \rrbracket^{C, s^{\prime}}=1$,

(ii) RESTRICTOR IF/USUALLY

$\llbracket$ if $p$, usually $q \rrbracket^{C, s}=1$ 
Operators or restrictors? A reply to Gillies

I argued above that the strategy Gillies (2010) pursues for epistemically modalized conditionals won't work as an extension of the context-shifty operator theory to adverbs of quantification: Closed neutralizes the epistemic quantification contributed by if and then Closed* neutralizes the epistemic quantification contributed by must. Thus, it remains unclear how to extend the context-shifty conditional operator story to predict the aforementioned facts about adverbially quantified conditionals. Furthermore, the problem isn't particular to adverbs of quantification - it arises for deontic modals as well.

\section{Deontic conditionals}

We can go back to our simple possible worlds semantics for now - we won't need situations here. Start with the following simple semantics for deontic necessity:

(36) $\llbracket$ have to $o_{D} p \rrbracket^{C, w}=1$ iff $\forall w \in \llbracket \mathrm{D} \rrbracket^{C, w}: \llbracket p \rrbracket^{C, w^{\prime}}=1$

(Where $\llbracket \mathrm{D} \rrbracket^{C, w}$ is the deontic modal base: the set of deontically ideal worlds given the $C$-relevant features of $w$.) The restrictor theory treats conditionals like if $p$, have $t o_{D} q$ by having the if-clause restrict the deontic modal, resulting in the following truth conditions:

(37) RESTRICTOR IF/HAVE $\mathrm{TO}_{D}$

$\llbracket$ if $p$, have to $q \rrbracket^{C, w}=1$ iff $\forall w^{\prime} \in\left(\llbracket \mathrm{D} \rrbracket^{C, w} \cap \llbracket p \rrbracket^{C}\right): \llbracket q \rrbracket^{C, w^{\prime}}=1$

If we focus on the O-reading of a particular deontic conditional like ${ }^{33}$

(38) If John harms someone, he has to be punished.

these truth conditions seem intuitively correct. They capture the sense in which (38) expresses a conditional obligation or command - in this case, an instance of some kind of retributivist principle: harmers must be punished. But notice that it's not sufficient for the truth of the O-reading of (38) that John in fact never harms someone, nor that there's some rule that would be satisfied if John is punished (regardless of whether he harmed someone).

33 I'm setting aside Geurts-complications for now - yes, these conditionals also seem to carry C-readings: see Geurts 2004: 8-10 for examples. Focus on the O-readings of the deontic conditionals in this section, which are the most natural readings without a lot of extra context. 
Rather, it seems to require for its truth that the ideal worlds (given some set of rules) in which John harms someone are all ones in which he is punished. This is just what the restrictor theory predicts, so predicting truth conditions equivalent to them (for at least this reading of deontic conditionals) is necessary for any adequate semantics of if.

Turning to Gillies' theory, I assume that $C+p$ affects deontic modal bases as it does epistemic modal bases:

$$
\llbracket \mathrm{D} \rrbracket^{C+p}==_{\operatorname{def}} \lambda w \cdot \llbracket \mathrm{D} \rrbracket^{C, w} \cap \llbracket p \rrbracket^{C}
$$

Following Gillies' strategy of scoping the modal underneath the conditional operator yields the following truth conditions for if $p$, have to ${ }_{D} q$ :

(40) GILliEs IF/HAVE $\mathrm{TO}_{D}$

$$
\begin{aligned}
& \llbracket \text { if } p \text {, have to } q \rrbracket^{C, w}=1 \text { iff } \forall w^{\prime} \in\left(\llbracket \mathrm{E} \rrbracket^{C, w} \cap \llbracket p \rrbracket^{C}\right): \\
& \forall w^{\prime \prime} \in\left(\llbracket \mathrm{D} \rrbracket^{C, w^{\prime}} \cap \llbracket p \rrbracket^{C}\right): \llbracket q \rrbracket^{C+p, w^{\prime \prime}}=1
\end{aligned}
$$

As with GiLlies If/UsUALLY, notice that the part underneath the universal quantifier over epistemically possible $p$-worlds is equivalent (assuming no modals in $q$ ) to the restrictor theory's truth conditions for if $p$, have to $_{D} q$. Hence, GILliEs IF/HAVE $\mathrm{TO}_{D}$ predicts that if $p$, have to $q$ requires for its truth that all $p$-worlds compatible with the relevant knowledge or evidence be ones at which the conditional obligation holds. These truth conditions are stronger than the restrictor theory's, which only require that the conditional obligation actually holds, and hence incorrect (since the restrictor theory's truth conditions are intuitively correct) for the O-reading: truth is one thing, certainty is another. ${ }^{34}$

The trouble here is the same as that facing Gillies' theory in the previous sections: to predict the O-reading of (38), the theory needs to neutralize the

34 Here's a scenario in which the RESTRICTOR IF/HAVE $\mathrm{TO}_{D}$ truth conditions are satisfied by the GILLIES IF/HAVE $\mathrm{TO}_{D}$ truth conditions are not. Suppose $\llbracket \mathrm{E} \rrbracket^{C, w}=\left\{w, w_{1}\right\}, \llbracket \mathrm{D} \rrbracket^{C, w}=\left\{w, w_{2}\right\}$, $\llbracket \mathrm{D} \rrbracket^{C+p, w_{1}}=\left\{w_{2}, w_{3}\right\}, \llbracket p \rrbracket^{C}=\left\{w, w_{1}, w_{2}, w_{3}\right\}$, and $\llbracket q \rrbracket^{C}=\left\{w, w_{2}\right\}$. Hence,

(i) $\quad \forall w^{\prime} \in\left(\llbracket \mathrm{D} \rrbracket^{C, w} \cap \llbracket p \rrbracket^{C}\right): \llbracket q \rrbracket^{C, w^{\prime}}=1$

and thus the RESTRICTOR IF/HAVE TO $_{D}$ truth conditions are satisfied. But,

(ii) $\quad \exists w^{\prime} \in\left(\llbracket \mathrm{E} \rrbracket^{C, w} \cap \llbracket p \rrbracket^{C}\right): \exists w^{\prime \prime} \in \llbracket \mathrm{D} \rrbracket^{C+p, w^{\prime}}: \llbracket q \rrbracket^{C+p, w^{\prime \prime}}=\mathrm{o}$

the witnessing world is $w_{1}$. Hence, the GILLIES IF/HAVE $\mathrm{TO}_{D}$ truth conditions are not satisfied on this scenario. 
Operators or restrictors? A reply to Gillies

epistemic quantification contributed by if when the deontic modal appears underneath it. As before, extending the strategy Gillies endorses in the case of epistemic modals would lead to adopting a principle that disallows differences in deontic modal bases across epistemically possible worlds:

(41) Closed $* *$

$$
\text { If } w^{\prime} \in \llbracket \mathrm{E} \rrbracket^{C, w} \text { then } \llbracket \mathrm{D} \rrbracket^{C, w}=\llbracket \mathrm{D} \rrbracket^{C, w^{\prime}}
$$

With this principle in hand, the truth conditions GILLIES IF/HAVE $\mathrm{TO}_{D}$ assigns to if $p$, must $_{D} q$ come out equivalent to those assigned by RESTRICTOR IF/HAVE $\mathrm{TO}_{D}$, as seems right ${ }^{35}$. But Closed $* *$ isn't plausible. For one, it doesn't seem to be entailed by any plausible features of epistemic or deontic modal bases. More importantly, GILLIES IF/MUST + Closed + Closed** predicts that conditionals of the form if $p$, must have $\mathrm{to}_{D} q$ are true just in case every deontically ideal $p$-world is a $q$-world (the proof is step by step the same as in the adverbial case - see fn 25). But this is false.

These truth conditions are incorrect for the same reason they are in the adverbial case: (the O-reading of) if $p$, have $t o_{D} q$ expresses a conditional deontic obligation, whereas if $p$, must $_{E}$ have $t_{D} q$ expresses a conditional epistemic necessity of a deontic obligation, but GILLIES IF/MUST + GILLIES IF $/$ HAVE $\mathrm{TO}_{D}+$ Closed + Closed $* *$ predicts that both express conditional obligations. However, distinguishing minimal pairs is made difficult by the fact that, in normal cases in which the former (conditional obligation) holds, the information that $p$ plus the available knowledge or evidence often entails have $t_{D} q$ (and hence the latter is true), and vice versa.

Nonetheless, there are cases in which $p$ plus the available knowledge or evidence doesn't entail have $t_{D} q$ even though the conditional obligation if $p$, have $t_{D} q$ holds. Suppose that, in John's household, chores are on a yearly cycle such that whoever handled the yard work last year has to wash dishes this year. In this situation it seems true that

(42) If John handled the yard work last year, he has to wash dishes this year.

Now, suppose your knowledge of the chore schedule is limited, and you only know enough to conclude, given that John handled the yard work last year, that he either has to do dishes or has to take out the garbage this year. In a context in which the only relevant knowledge is yours, the following would

35 The proof is exactly analogous to the proof in the adverbial case. 
Justin Khoo

be false:

(43) If John handled the yard work last year, he must have to wash dishes this year.

since the following is true (in this context):

(44) If John handled the yard work last year, he might have to take out the garbage this year.

Therefore, it's not true, as predicted by GILLIES IF/MusT + Closed + Closed $* *$, that if $p$, must $_{E}$ have to $q$ is true iff every deontically ideal $p$-world is a $q$-world. ${ }^{36}$

As with adverbially quantified conditionals, deontic conditionals of the form if $p$, have $t_{D} q$ carry O-readings which are true iff every deontically ideal $p$-world is a $q$-world, whereas it is not the case that conditionals of the

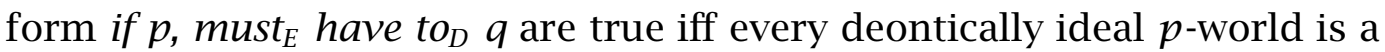
$q$-world. To predict both facts, Gillies' theory needs a way to neutralize the epistemic quantification contributed by if when a deontic modal falls in its scope while not neutralizing the epistemic quantification contributed by the must which scopes between the conditional operator and the deontic modal in conditionals of the form

(45) if $p$, must $_{E}$ MODAL $_{D} q$

As with adverbially quantified conditionals, the strategy Gillies (2010) pursues for epistemically modalized conditionals won't work as an extension of the context-shifty operator theory to deontic modals: Closed neutralizes the epistemic quantification contributed by if and then Closed** neutralizes the epistemic quantification contributed by must $_{E}$. It remains unclear how to extend the context-shifty conditional operator story to predict the aforementioned facts about deontic conditionals.

$36 \overline{\text { More formally, suppose that } \llbracket \mathrm{D} \rrbracket^{C, w}} \cap \llbracket$ John handled the yard work last year $\rrbracket^{C}=\{j\}$ and that $\llbracket$ John washes dishes this year $\rrbracket^{C, j}=1$. Then, $\forall w^{\prime} \in\left(\llbracket \mathrm{D} \rrbracket^{C, w} \cap \llbracket\right.$ John handled the yard work last year $\rrbracket^{C}$ ): $\llbracket$ John washes dishes this year $\rrbracket^{C, w^{\prime}}=1$ and hence (42) is true. Next, suppose that $\llbracket \mathrm{E} \rrbracket^{C, w} \cap \llbracket$ John handled yard work last year $\rrbracket^{C}=\{k, l\}$ and $\llbracket$ John has to wash dishes this year $\rrbracket^{C, k}=0$. Then, $\exists w^{\prime} \in\left(\llbracket \mathrm{E} \rrbracket^{C, w} \cap \llbracket\right.$ John handled yard work last year $\left.\rrbracket^{C}\right): \llbracket$ John has to wash dishes this year $\rrbracket^{C, w^{\prime}}=0$, and thus the relevant knowledge plus the assumption that John did the yard work last year does not entail that John has to wash dishes this year. Hence, (43) is intuitively false in this scenario. 
Operators or restrictors? A reply to Gillies

\section{Conclusion}

Here's the moral of the story. Conditional operator theories build the distinctive meaning of bare conditionals into the operator denoted by if. The quantification contributed by this conditional operator must be neutralized when another quantifier appears in the consequent of the conditional, even though the information of the if-clause remains relevant for the evaluation of that quantifier - at least, the O-readings of quantified conditionals demand this much. Gillies' shifty operator theory, in which if is a strict conditional over epistemic possibilities whose consequent is evaluated in a if-shifted subordinate context, appears to meet both demands for conditionals with epistemic modals in their consequents, given a plausible principle that collapses stacked epistemic modals. But when we generalize the theory to handle conditionals with adverbial quantifiers or deontic modals in their consequents, a major problem emerges.

The problem is that we can stack an epistemic modal on top of an adverbial quantifier or deontic modal appearing in the consequent of a conditional, and doing so has truth conditional consequences that a natural extension of Gillies' theory - one that gets the O-readings of adverbially quantified and deontic conditionals by appealing to analogous principles to the one used to get the right truth conditions of epistemically modalized conditionals - doesn't predict. That is, the natural extension involves assuming Closed*/Closed** to neutralize the epistemic quantification contributed by if when an adverb of quantification or deontic modal scopes underneath it and hence predict the O-readings of
a. if $p, \operatorname{ADVQ} q$
b. if $p, \operatorname{MODAL}_{D} q$

But assuming these principles in addition to the original principle Gillies' theory uses to collapse stacked epistemic modals (Closed) results in incorrect truth conditions assigned to conditionals of the form
(47)
a. if $p$, must $_{E}$ ADVQ $q$
b. if $p$, must $_{E}$ MODAL $_{D} q$

Thus, although the strategy for neutralizing the epistemic quantification contributed by if when an epistemic modal scopes under it seems plausible, analogous strategies are not available for the context-shifty theory in the 
Justin Khoo

adverbial or deontic realm. ${ }^{37}$ This is not to say that no context-shifty conditional operator could do the trick, but as of now, there is no promising blueprint for doing so.

\section{References}

Beaver, David \& Brady Clark. 2008. Sense and sensitivity: How focus determines meaning. Oxford: Blackwell.

Berman, Stephen. 1987. Situation-based semantics for adverbs of quantification. In James Blevins \& Anne Vainikka (eds.), Studies in semantics (University of Massachusetts Occasional Papers in Linguistics (UMOP) 12), 46-68. Amherst: GLSA, University of Masschusetts at Amherst.

Chierchia, Gennaro. 1992. Anaphora and dynamic binding. Linguistics and Philosophy 15(2). 111-183. doi:10.1007/BFoo635805.

Elbourne, Paul D. 2005. Situations and individuals. Cambridge: MIT Press.

Farkas, Donka \& Yoko Sugioka. 1983. Restrictive if/when clauses. Linguistics and Philosophy 6(2). 225-258. doi:10.1007/BFoo635644.

von Fintel, Kai. 1994. Restrictions on quantifier domains. Amherst: University of Massachusetts at Amherst dissertation. http://semanticsarchive.net/ Archive/jA3N2IwN/fintel-1994-thesis.pdf.

von Fintel, Kai. 2004. A minimal theory of adverbial quantification. In Hans Kamp \& Barbara Partee (eds.), Context-dependence in the analysis of linguistic meaning, 153-193. Amsterdam: Elsevier.

von Fintel, Kai \& Anthony Gillies. 2010. Must ... stay ... strong! Natural Language Semantics 18(4). 351-383. doi:10.1007/s11050-010-9058-2.

Geurts, Bart. 2004. On an ambiguity in quantified conditionals. http://ncs. ruhosting.nl/bart/papers/conditionals.pdf.

Gillies, Anthony. 2010. Iffiness. Semantics \& Pragmatics 3(4). 1-42. doi:10.3765/sp.3.4.

Heim, Irene. 1982. The semantics of definite and indefinite noun phrases.

37 One way to "neutralize" the iffy epistemic quantification is to hold if doesn't contribute a conditional operator at all when it combines with adverbs of quantification and deontic modals. This strategy follows Lewis's lead by holding that if is ambiguous and that one of its denotations is a restrictor device. But adopting such a strategy at this point in the dialectic seems confused (not to mention out of step with Gillies' own assumption of univocality - see fn 2), since it undercuts the motivation for the context-shifting operator in the first place. Since we could just as well invoke this strategy to handle bare and epistemically modalized conditionals to begin with, what would the motivation be for treating them but not adverbially quantified or deontic conditionals, uniformly? 
Operators or restrictors? A reply to Gillies

Amherst: University of Massachusetts at Amherst dissertation. http: //semanticsarchive.net/Archive/TkoZmYyY/dissertation.pdf.

Heim, Irene. 1990. E-type pronouns and donkey anaphora. Linguistics and Philosophy 13(2). 137-177. doi:10.1007/BFoo630732.

Kadmon, Nirit. 1987. On unique and non-unique reference and asymmetric quantification: University of Massachusetts at Amherst dissertation.

Kamp, Hans. 1981. A theory of truth and semantic interpretation. In Jeroen Groenendijk, Theo Janssen \& Martin Stokhof (eds.), Formal methods in the study of language: Part I, 277-322. Amsterdam: Mathematisch Centrum.

Kratzer, Angelika. 1986. Conditionals. Chicago Linguistics Society 22(2). 1-15.

Kratzer, Angelika. 1989. An investigation of the lumps of thought. Linguistics and Philosophy 12(5). 607-653. doi:10.1007/BFoo627775.

Kratzer, Angelika. 1991. Modality. In Arnim von Stechow \& Dieter Wunderlich (eds.), Semantics: An international handbook of contemporary research, 639-650. Berlin and New York: de Gruyter.

Kratzer, Angelika. 2011a. Collected papers on modals and conditionals. Oxford: Oxford University Press.

Kratzer, Angelika. 2011b. Situations in natural language semantics. In Edward N. Zalta (ed.), The Stanford encyclopedia of philosophy, http: //plato.stanford.edu/archives/fall2011/entries/situations-semantics/.

Krifka, Manfred, Francis Jeffry Pelletier, Greg Carlson, Alice ter Meulen, Gennaro Chierchia \& Godehard Link. 1995. Genericity: an introduction. In The generic book, 1-124. Chicago: The University of Chicago Press.

Lewis, David. 1973. Counterfactuals. Oxford: Blackwell.

Lewis, David. 1975. Adverbs of quantification. In Edward L. Keenan (ed.), Formal semantics of natural language, 3-15. Cambridge: Cambridge University Press.

Lewis, David. 1976. Probabilities of conditionals and conditional probabilities. The Philosophical Review 85(3). 297-315. doi:10.2307/2184045.

Portner, Paul. 2009. Modality (Oxford Surveys in Semantics and Pragmatics 1). Oxford: Oxford University Press.

Yalcin, Seth. 2007. Epistemic modals. Mind 116(464). 983-1026. doi:10.1093/mind/fzmg83.

Justin Khoo

Department of Philosophy

Yale University

justin.khoo@yale.edu 